The meta-analysis included 10 studies $(n=476)$. The decline in proteinuria over a median follow-up period of 6 months was compared between patients given pentoxifylline (400-1,200 mg per day) and control patients who received placebo or standard care. Angiotensin-converting-enzyme inhibitors or angiotensin receptor blockers were also administered to both groups in four of the studies and to only the control group in two of the studies. Overall, the decrease in proteinuria associated with pentoxifylline was greater than the decrease associated with placebo or standard care (weighted mean difference $-278 \mathrm{mg}$ per day, $95 \% \mathrm{Cl}-398$ to $-159 \mathrm{mg}$ per day; $P<0.001$ ). Subanalyses based on baseline proteinuria indicated that use of pentoxifylline was associated with a significant decrease in proteinuria compared with placebo or standard care in patients with overt proteinuria $(n=210$; weighted mean difference $-502 \mathrm{mg}$ per day, $95 \% \mathrm{Cl}-805$ to $-198 \mathrm{mg}$ per day; $P=0.001)$. In patients with microalbuminuria $(n=104)$, a nonsignificant decrease in proteinuria was observed with pentoxifylline compared with placebo or standard care. Seven adverse events occurred in patients who received pentoxifylline. No significant difference was noted between the effects of pentoxifylline and placebo or standard care on either serum creatinine or blood pressure levels.

The authors conclude that pentoxifylline, which is relatively inexpensive, could be particularly helpful in decreasing proteinuria in patients with diabetic nephropathy, in whom angiotensin-converting-enzyme inhibitors or angiotensin receptor blockers cannot be used.

Original article McCormick BB et al. (2008) The effect of pentoxifylline on proteinuria in diabetic kidney disease: a meta-analysis. Am J Kidney Dis [doi:10.1053/ j.ajkd.2008.01.025]

\section{Hypertensive CKD in African Americans progresses despite treatment}

The number of patients with hypertensive chronic kidney disease (CKD) who reach end-stage renal disease is disproportionately higher among African Americans than among whites, yet African Americans have been under-represented in studies of CKD. Appel et al. have determined that in most African Americans with CKD and hypertension, CKD progresses even when treated according to current guidelines - only approximately a third of patients do not progress or progress slowly.

In the African American Study of Kidney Disease and Hypertension, which took place from April 1995 to September 2001, African Americans with hypertensive CKD were randomly allocated to a 'normal' $(102-107 \mathrm{mmHg})$ or 'low' $(<92 \mathrm{mmHg})$ mean arterial pressure goal, and to receive a reninangiotensin-system blocker, a $\beta$-blocker, or a calcium-channel blocker. In all, 647 patients subsequently participated in a cohort study (from April 2002 to June 2007) in which they were assigned a blood pressure goal according to prevailing guidelines (mean achieved blood pressure $133 / 78 \mathrm{mmHg}$ ). Most patients received renin-angiotensin-system-blocking medication. Taking both studies into account, 576 (52\%) of the 1,094 participants experienced the primary outcome-doubled serum creatinine level from baseline, end-stage renal disease or deathcorresponding to a 10-year cumulative event rate of $53.9 \%$.

The authors suggest that current guidelines do not need to be changed, but they highlight the critical need for additional research on the determinants of CKD progression in this population.

Original article Appel LJ et al. (2008) Long-term effects of renin-angiotensin system-blocking therapy and a low blood pressure goal on progression of hypertensive chronic kidney disease in African Americans. Arch Intern Med 168: 832-839

\section{Risk of death versus end-stage renal disease in patients with CKD without diabetes}

The few studies that have investigated the long-term risks of end-stage renal disease (ESRD) and death in patients with chronic kidney disease (CKD) have found that mortality, which is usually due to cardiovascular events, is a more likely outcome than ESRD. These results might, however, have been influenced by the studies' focus on subgroups of patients with CKD in non-CKD cohorts, older patients with early-stage CKD, or populations with a high prevalence of diabetes.

Menon et al. retrospectively evaluated the outcomes of 1,666 patients with stage $2-4$ 